\title{
Flow of a viscoelastic fluid over a stretching sheet
}

\author{
K. R. Rajagopal, T. Y. Na, and A. S. Gupta \\ Department of Mechanical Engineering, University of Pittsburgh, \\ Department of Mechanical Engineering, University of Michigan at Dearborn, and \\ Department of Mathematics, Indian Institute of Technology at Kharagpur
}

\begin{abstract}
This paper presents a study of the flow of an incompressible second-order fluid past a stretching sheet. The problem has a bearing on some polymer processing application such as the continuous extrusion of a polymer sheet from a die.
\end{abstract}

Key words: Second-order fluid, stretching sheet, similarity solution

\section{Introduction}

The flow in the boundary layer of an incompressible viscous fluid on moving solid surfaces has been investigated by Sakiadis [1]. Due to the entrainment of the ambient fluid, this boundary layer is quite different from that in Blasius flow past a flat plate. Erickson, Fan and Fox [2] extended this problem to the case in which the transverse velocity at the moving surface is non-zero, with heat and mass transfer in the boundary layer being taken into account. These investigations have a bearing on the problem of a polymer sheet extruded continuously from a die. It is often tacitly assumed that the sheet is inextensible, but situations may arise in the polymer industry in which it is necessary to deal with a stretching plastic sheet, as pointed out by McCormack and Crane [3]. Danberg and Fansler [4] investigated the non-similar solution for the flow in the boundary layer past a wall that is stretched with a velocity proportional to the distance along the wall, the free-stream velocity being constant. Gupta and Gupta [5] analysed the heat and mass transfer corresponding to the similarity solution for the boundary layer over a stretching sheet subjected to suction or blowing.

All the above investigations were restricted to flows of Newtonian fluids. However, of late non-Newtonian fluids have become more and more important industrially. The laminar boundary layer on an inextensible continuous flat surface moving with a constant velocity in its own plane in a non-Newtonian fluid characterized by a power-law model (Ostwald-de Waele fluid) was studied by Fox, Erickson and Fan [6] using both exact 947 and approximate methods. Apart from the limitations of the above power-law model, which does not exhibit any elastic properties (such as normal-stress differences in shear flow), in certain polymer processing applications one deals with flows of a viscoelastic fluid over a stretching sheet. This provides the motivation for the present study in which the flow of a class of viscoelastic fluids past a stretching sheet is examined. These are the incompressible second-order fluids whose constitutive equation based on the postulate of gradually fading memory was given by Coleman and Noll [7] as

$$
\boldsymbol{T}=-p \boldsymbol{l}+\mu \boldsymbol{A}_{1}+\alpha_{1} \boldsymbol{A}_{2}+\alpha_{2} \boldsymbol{A}_{1}^{2},
$$

where $\boldsymbol{T}$ is the stress tensor, $p$ the pressure, $\mu, \alpha_{1}, \alpha_{2}$ are material constants with $\alpha_{1}<0$, and $\boldsymbol{A}_{1}$ and $\boldsymbol{A}_{2}$ are defined as

$$
\begin{aligned}
& \boldsymbol{A}_{1}=(\operatorname{grad} v)+(\operatorname{grad} v)^{\mathrm{T}}, \\
& \boldsymbol{A}_{2}=\frac{d}{d t} \boldsymbol{A}_{1}+\boldsymbol{A}_{1} \cdot \operatorname{grad} \boldsymbol{v}+(\operatorname{grad} v)^{\mathrm{T}} \cdot \boldsymbol{A}_{1} .
\end{aligned}
$$

Coleman and Noll showed that the model (1.1) exhibits normal-stress differences in shear flow and is an approximation to a simple fluid in the sense of retardation. This model is applicable to some dilute polymer solutions (such as the 5.4 percent solution of polyisobutylene in cetane reported by Markovitz and Coleman [8]) at low rates of shear.

\section{Calculation}

We consider the flow of a second-order fluid obeying (1.1) past a wall coinciding with the plane $y=0$, the 
flow being confined to $y>0$. Two equal and opposite forces are applied along the $x$-axis so that the wall is stretched whilst keeping the origin fixed (figure 1).

The steady two-dimensional boundary layer equations for this fluid were derived by Beard and Walters [9]. In usual notation these equations are

$$
\begin{aligned}
& \frac{\partial u}{\partial x}+\frac{\partial v}{\partial y}=0 \\
& u \frac{\partial u}{\partial x}+v \frac{\partial u}{\partial y} \\
& \quad=v \frac{\partial^{2} u}{\partial y^{2}}-k\left[\frac{\partial}{\partial x}\left(u \frac{\partial^{2} u}{\partial y^{2}}\right)+\frac{\partial u}{\partial y} \frac{\partial^{2} v}{\partial y^{2}}+v \frac{\partial^{3} u}{\partial y^{3}}\right]
\end{aligned}
$$

where

$$
\nu=\mu / \varrho, \quad k=-\alpha_{1} / \varrho .
$$

In deriving these equations it was assumed that in addition to the usual boundary layer approximations the contribution due to the normal stresses is of the same order of magnitude as that due to the shear stresses. Thus both $v$ and $k$ are $O\left(\delta^{2}\right), \delta$ being the boundary layer thickness.

The boundary conditions are

$$
\begin{aligned}
& u=c x, \quad v=0 \text { at } y=0 \\
& u \rightarrow 0 \text { as } y \rightarrow \infty, \quad c>0 .
\end{aligned}
$$

The flow is caused solely by the stretching of the wall, the free stream velocity being zero. Eqs. (2.1) and (2.2) have the similarity solution

$$
u=c x f^{\prime}(\eta), \quad v=-(v c)^{1 / 2} f(\eta)
$$

where the similarity variable $\eta$ is given by

$$
\eta=(c / v)^{1 / 2} y
$$

Clearly $u$ and $v$ given in (2.5) satisfy (2.1), and substituting into (2.2) gives

$$
f^{\prime 2}-f f^{\prime \prime}=f^{\prime \prime \prime}-k_{1}\left[2 f^{\prime} f^{\prime \prime \prime}-\left(f^{\prime \prime}\right)^{2}-f f^{\mathrm{iv}}\right] \text {, }
$$

where a prime denotes differentiation with respect to $\eta$

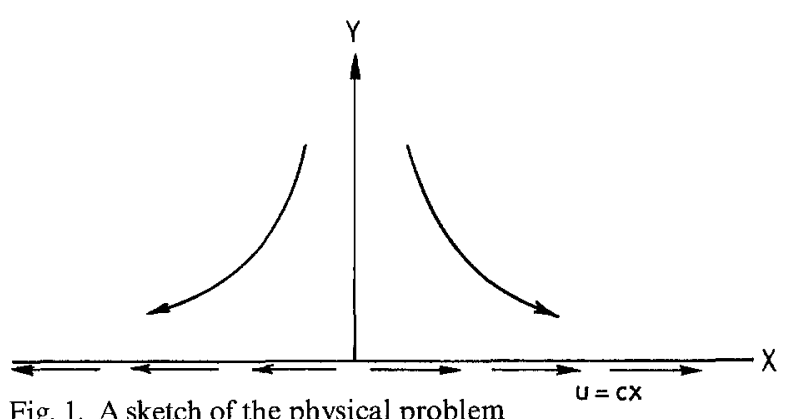

Fig. 1. A sketch of the physical problem and

$$
k_{1}=k c / v \text {. }
$$

The boundary conditions (2.4) are transformed to

$$
f^{\prime}(0)=1, \quad f(0)=0, \quad f^{\prime}(\infty)=0 \text {. }
$$

Eq. (2.7) is an equation of fourth order with the three boundary conditions (2.9). To overcome this difficulty (which essentially involves a singular perturbation problem) we follow Beard and Walters [9] and assume $k_{1}$ to be small. Since a second-order fluid obeying (1.1) represents the behaviour of fluids with short memory (Craik [10]) and the characteristic time scale associated with the motion is large compared with the time $\left|\alpha_{1} / \mu\right|$ representing the memory of the fluid, the assumption of small $k_{1}$ is valid, in particular, for dilute polymer solutions.

Hence, we expand $f$ in a power series as

$$
f=f_{0}+k_{1} f_{1}+k_{1}^{2} f_{2}+\ldots
$$

Substituting (2.10) in (2.7) and equating the coefficients of $k_{1}^{0}$ and $k_{1}$ results in

$$
\begin{aligned}
& {f_{0}}^{\prime 2}-f_{0} f_{0}^{\prime \prime}=f_{0}^{\prime \prime \prime}, \\
& f_{1}^{\prime \prime \prime}+f_{0} f_{1}^{\prime \prime}+f_{0}^{\prime \prime} f_{1}-2 f_{0}^{\prime} f_{1}^{\prime}=s,
\end{aligned}
$$

with

$$
s=2 f_{0}^{\prime} f_{0}^{\prime \prime \prime}-f_{0}^{\prime \prime 2}-f_{0} f_{0}^{\mathrm{iv}} .
$$

Using (2.9), the boundary conditions for $f_{0}$ and $f_{1}$ are

$$
\begin{aligned}
& f_{0}^{\prime}(0)=1, \quad f_{0}(0)=0, \quad f_{0}^{\prime}(\infty)=0, \\
& f_{1}^{\prime}(0)=0, \quad f_{1}(0)=0, \quad f_{1}^{\prime}(\infty)=0 .
\end{aligned}
$$

The solution of (2.11) satisfying (2.14) is

$$
f_{0}(\eta)=1-\mathrm{e}^{-\eta}
$$

For solving (2.12), which is linear in $f_{1}(\eta)$, we assume

$$
f_{1}=f_{A}+\beta_{1} f_{B}
$$

such that

$$
\begin{aligned}
& f_{A}^{\prime \prime \prime}+f_{0} f_{A}^{\prime \prime}-2 f_{0}^{\prime} f_{A}^{\prime}+f_{0}^{\prime \prime} f_{A}=s, \\
& f_{B}^{\prime \prime \prime}+f_{0} f_{B}^{\prime \prime}-2 f_{0}^{\prime} f_{B}^{\prime}+f_{0}^{\prime \prime} f_{B}=0,
\end{aligned}
$$

with the boundary conditions [see (2.15)]

$$
\begin{aligned}
& f_{A}(0)=f_{A}^{\prime}(0)=0, \quad f_{A}^{\prime \prime}(0)=0, \\
& f_{B}(0)=f_{B}^{\prime}(0)=0, \quad f_{B}^{\prime \prime}(0)=1 .
\end{aligned}
$$

Eqs. (2.18) and (2.19) are then integrated numerically using the Runge-Kutta method and the boundary conditions (2.20) and (2.21). To determine the param- 
eter $\beta_{1}$ in $(2.17)$, we use the third boundary condition in (2.15) which gives

$$
\beta_{1}=-\frac{f_{A}^{\prime}(\infty)}{f_{B}^{\prime}(\infty)}
$$

Using (1.1), (2.5) and (2.9), the dimensionless shear stress $\tau$ at the wall is given by

$$
\tau=x^{-1} v^{-1 / 2} c^{-3 / 2}\left(T_{x y}\right)_{y=0}=\left(1-k_{1}\right) f^{\prime \prime}(0) \text {. }
$$

\section{Results}

The following table gives the values of $\tau$ for several values of $k_{1}$.

Table 1. Values of $\tau$

\begin{tabular}{lc}
\hline$k_{1}$ & $\tau$ \\
\hline 0.005 & -0.9975 \\
0.01 & -0.9949 \\
0.03 & -0.9846 \\
0.05 & -0.9738 \\
\hline
\end{tabular}

It is seen that the magnitude of the skin-friction coefficient decreases with increasing values of the elastic parameter $k_{1}$. For industrial applications, this result is of some importance since the power expenditure involved in stretching the sheet decreases with increasing $k_{1}$.

Table 2 gives the variation of $f^{\prime}(\eta)$ with $\eta$ for several values of $k_{1}$.

Table 2. Values of $f^{\prime}(\eta)$

$\begin{array}{lllllllll}\eta & & & & & & & & \\ k_{1}\end{array}$

$\begin{array}{lllllllll}0.005 & 0.7535 & 0.5241 & 0.3290 & 0.0842 & 0.0090 & 0.0018 & 0.0002\end{array}$

$\begin{array}{lllllllllll}0.01 & & 0.7529 & 0.5232 & 0.3280 & 0.0837 & 0.0089 & 0.0017 & 0.0002\end{array}$

$\begin{array}{lllllllll}0.05 & & 0.7487 & 0.5164 & 0.3205 & 0.0795 & 0.0080 & 0.0015 & 0.0001\end{array}$

Since $u$ is proportional to $f^{\prime}(\eta)$ [see (2.5)] it follows that $u$ decreases as $k_{1}$ increases. Thus the boundary layer thickness increases as the value of the parameter $k_{1}$ increases, which might be regarded as a manifesta- tion of the presence of normal stresses inside the boundary layer. In fact, the physical explanation of the thickening of the boundary layer may be attributed to tensile stresses in the layer which cause an axial contraction and hence thickening of the layer in the transverse direction.

It may be noticed that the cross-viscosity coefficient $\alpha_{2}$ does not affect the velocity distribution since the flow is two-dimensional although $\alpha_{2}$ affects the pressure distribution.

\section{Acknowledgement}

K R. Rajagopal would like to thank the Macromolecular Research Institute at the University of Michigan for its support.

\section{References}

1. Sakiadis BD (1961) AICHE J 7:26

2. Erickson LE, Fan LT, Fox VG (1966) Ind Eng Chem Fundam 5:19

3. McCormack PD, Crane L (1973) Physical Fluid Dynamics, Academic Press, New York

4. Danberg JE, Fansler KS (1976) Quart Appl Math 34:305

5. Gupta PS, Gupta AS (1977) Canad J Chem Eng 55:744

6. Fox VG, Erickson LE, Fan LT (1969) AIChE J 15:327

7. Coleman BD, Noll W (1960) Arch Rational Mech Anal 6:355

8. Markovitz H, Coleman BD (1964) Adv Appl Mech, Vol 8 (Academic Press)

9. Beard DW, Waters K (1964) Proc Camb Phil Soc 60:667

10. Craik ADD (1968) J Fluid Mech 33:33

(Received March 7, 1983)

Authors' addresses:

Dr. K. R. Rajagopal

Department of Mechanical Engineering

University of Pittsburgh

Pittsburgh, PA 15261 (USA)

Dr. T. Y. Na

Department of Mechanical Engineering

University of Michigan

Dearborn, MI 48128 (USA)

Prof. A. S. Gupta

Department of Mathematics

Indian Institute of Technology

Kharagpur 721302 (India) 